\title{
IDENTIFICATION OF AQUIFER TRANSMISSIVITY WITH MULTIPLE SETS OF DATA USING THE DIFFERENTIAL SYSTEM METHOD
}

\author{
M. Giudici, ${ }^{1}$ G. A. Meles, ${ }^{1}$ G. Parravicini, ${ }^{2}$ G. Ponzini, ${ }^{1}$ and C. Vassena ${ }^{1}$ \\ ${ }^{1}$ Università degli Studi di Milano, Dipartimento di Scienze della Terra, Sezione di Geofisica, \\ via Cicognara 7, Milano, Italy, \{Mauro.Giudici, Giansilvio.Ponzini,Chiara.Vassena\}@unimi.it \\ ${ }^{2}$ Università degli Studi di Milano, Dipartimento di Fisica, via Celoria 16, I-20133 Milano, Llaly, \\ Guido.Parravicini@unimi.it
}

\begin{abstract}
The mass balance equation for stationary flow in a confined aquifer and the phenomenological Darcy's law lead to a classical elliptic PDE, whose phenomenological coefficient is transmissivity, $T$, whereas the unknown function is the piezometric head. The differential system method (DSM) allows the computation of $T$ when two "independent" data sets are available, i.e., a couple of piezometric heads and the related source or sink terms corresponding to different flow situations such that the hydraulic gradients are not parallel at any point. The value of $T$ at only one point of the domain, $\mathbf{x}_{0}$, is required. The $T$ field is obtained at any point by integrating a first order partial differential system in normal form along an arbitrary path starting from $\mathrm{x}_{0}$. In this presentation the advantages of this method with respect to the classical integration along characteristic lines are discussed and the DSM is modified in order to cope with multiple sets of data. Numerical tests show that the proposed procedure is effective and reduces some drawbacks for the application of the DSM.
\end{abstract}

keywords: Inverse problems, porous media, multiple data sets

\section{Problem definition and classical methods of solution}

We consider ground water flow in a confined aquifer, i.e. a permeable porous geological formation with upper and lower impermeable boundaries. The mass balance equation for stationary flow (which means that the fluid density is constant and the porous medium is not deforming), can be written as

$$
\partial_{x}\left(T \partial_{x} h\right)+\partial_{y}\left(T \partial_{y} h\right)=f
$$

where $T$ is the aquifer transmissivity $\left[L^{2} / T\right], h$ is the piezometric head $[L]$ and $f$ is the source term, i.e. the well discharge rate of abstracted water per unit area of the aquifer $[L / T]$. The development of a forecasting model requires the solution to (1) with respect to $h$, so that $T$ and $f$ must be known.

Please use the following format when citing this chapter:

Giudici, M., Meles, G.A.., Parravicini, G., Ponzini, G., and Vassena, C., 2006, in IFIP International Federation for Information Processing, Volume 202, Systems, Control, Modeling and Optimization, eds. Ceragioli, F., Dontchev, A., Furuta, H., Marti, K., Pandolfi, L., (Boston: Springer), pp. 175-181. 
Data on $T$ are usually obtained from the interpretation and processing of well tests, which are very much influenced by the well characteristics (head losses due to screen and drain effect, pump position, etc.) and provide a value which can be representative of a region with a limited radius around the well, say of the same order of magnitude of the screened intervals, which could be of the order of tens of meters. As a consequence, these values are not representative of the flow processes at a regional scale, where flow is modelled in aquifers whose lateral extensions could be as great as tens or hundreds of kilometers and for which the spacing of the numerical grid could be hundreds of meters.

The $T$ field has to be estimated, for example with the solution of an inverse problem for equation (1). This requires the computation of $T$, given $h$ and $f$ and the least prior knowledge of $T$.

In the mathematical and geophysical literature this inverse problem has been classically posed as a Cauchy problem, and the solution is found by integration along the flow lines (see, e.g., [8], [9], [10], [2], [3], [13]). For this it is necessary to assign $T$ at a point for each flow line. The application of such an approach to real cases is very difficult, practically impossible. In fact it is difficult to measure $T$ along the inflow or outflow boundary of the domain or wherever at a point along each flow line. It is also difficult to determine the flow lines with enough precision from head data which are available at a limited number of irregularly scattered points. Moreover, since the $T$ field depends upon the hydraulic gradient, grad $h$, the integration of (1) with respect to $T$ along a flow line is intrinsically unstable. Since the integration along each flow path is independent from the integration along the neighbouring flow lines, the instability could lead to results which do not respect any regularity of the $T$ field among nearby flow lines (see [3] for a discussion about practical aspects).

Other approaches, related to non linear least-squares techniques, possibly with regularization, or in the framework of maximum likelihood estimation, assume some knowledge of the unknown parameter [18], [1], e.g., the fact that it is piecewise constant so that the domain can be partitioned into a number of subdomains where $T$ is constant. This approach is known as zonation.

Instead of using additional prior information on $T$, which always poses problems of data effectiveness, other methods can reduce the above mentioned problem for inversion through the use of data measured at different times and therefore related to different flow situations. See [15], [14], [12], [1], [4], [16], [19], [7] among the others.

The next section describes one of these methods, the Differential System Method (DSM). 


\section{The Differential System Method}

The simplest version of the DSM, see [5] and [11], allows for a solution of the inverse problem when two independent sets of data, $\left\{\left(h^{(l)}, f^{(l)}\right), l=1,2\right\}$, and the value of $T$ at only one point $\mathrm{x}_{0}$ of the domain are available. In this case equation (1) can be written for both data sets and leads to a system of first order partial differential equations for $T$, which can be written in the normal form

$$
\operatorname{grad} T=-T \mathbf{a}+\mathbf{b}
$$

if the following independence condition holds:

$$
\operatorname{det} A \neq 0,
$$

where the elements of $A$ are given by the relations

$$
A_{i, l}=\partial_{i} h^{(l)}
$$

The $T$ field is obtained at any point $\mathrm{x}$ by integration of the differential equation (2) in the unknown function $T$ along any line connecting $\mathrm{x}$ to $\mathrm{x}_{0}$, where the value, $T_{0}$, of $T$ at $\mathrm{x}_{0}$ is the initial value for the integration. The integration path, $\gamma$, can be chosen according to a stability condition that requires that the line integral $\int_{\gamma}|\mathbf{a}| d l$ be small in order that the error propagation along the integration line $\gamma$ be small.

The DSM has been tested with stationary [5] and transient [17] synthetic data. A discussion on the discrete stability of the method is given in [5] and numerical experiments are shown in [6].

The numerical tests so far performed show that the stability condition is important also for the choice of the starting point $\mathrm{x}_{0}$. In fact if $\mathrm{x}_{0}$ is chosen in an area where $|\mathbf{a}|$ is great, numerical errors prevent the computation of $T$ with a good confidence but for a small neighborhood of $\mathrm{x}_{0}$. Unfortunately the data on $T$ are usually available where well tests can be performed; as a consequence, $\mathrm{x}_{0}$ should correspond to the location of an existing well where tests have been performed and nobody can guarantee that $|\mathbf{a}|$ is small there.

Another difficulty for the application of the DSM to real cases is the fact that data sets independent on the whole domain can be obtained for a variation of the physical boundary conditions, which is nevertheless quite rare and above all cannot be controlled. In fact boundary conditions vary as a response to climate change, modification of land use, and so on. On the other hand, a variation of the pumping schedule modifies the flow field in limited regions surrounding the pumping wells only and not throughout the whole aquifer (see, e.g., [16]). 


\section{The Differential System Method with multiple sets of data}

The difficulties discussed at the end of the previous section might be mitigated if the DSM is modified in order to deal with several sets of data, i.e. $M$ pairs $\left(h^{(l)}, f^{(l)}\right), l=1, \ldots, M$, with $M>2$. Equation (1) can be written for all the available data sets. The standard version of the DSM can be applied if we locally choose the "best" pair of sets of data to build the matrix A, as defined by (4), and compute the vectors $\mathbf{a}$ and $\mathbf{b}$ to be used in (2).

In particular multiple data sets can be used pairwise to compute the vectors $\mathbf{a}$ and $\mathbf{b}$ in the following way. The domain is subdivided in subregions, where a pair of data sets can be found that best satisfies the following conditions:

1. the independence condition;

2. the stability condition;

3. the smallness of $\mu(A)$, the condition number of $A$.

In particular, $\mu(A)$ is computed as follows

$$
\mu(A)=\|A\| \cdot\left\|A^{-1}\right\|=\frac{\sum_{i, j} a_{i, j}^{2}}{|\operatorname{det} A|},
$$

where the Frobenius norm is used. Once the vectors $\mathbf{a}$ and $\mathbf{b}$ have been computed with the "best" pair of sets, the DSM can be applied with the standard procedure in each subregion.

\section{Numerical tests}

In this section some results of simple numerical tests are shown. More complex cases have also been analysed, but the results are qualitatively very similar, so that this simple case could be more easy to be analysed and interpreted.

The reference $\log (T)$ field is represented in figure 1, together with the position of the abstraction wells that are used to generate the synthetic head data.

In particular one set of data (set 0 ) is obtained with no pumping wells and nine data sets (sets 1 to 9) correspond to the cases when one well at time is pumping, with the discharge rates (in $\mathrm{L} / \mathrm{s}$ ) plotted in figure 1. For each data set the noise-free head data are obtained with a finite difference solution of the discrete balance equation; the assigned Dirichlet boundary conditions are linearly varying from left $(100 \mathrm{~m})$ to right $(80 \mathrm{~m})$. Then the data are corrupted with an uncorrelated noise. Here we show the results when the noise is introduced with a truncation of the piezometric heads at the third decimal digit.

The results of the standard DSM applied to the data sets 0 and 4 are shown in figure 2 when the starting point is at well no. 1 or 9 . The differences between the two cases are apparent. In particular when the starting point corresponds to 


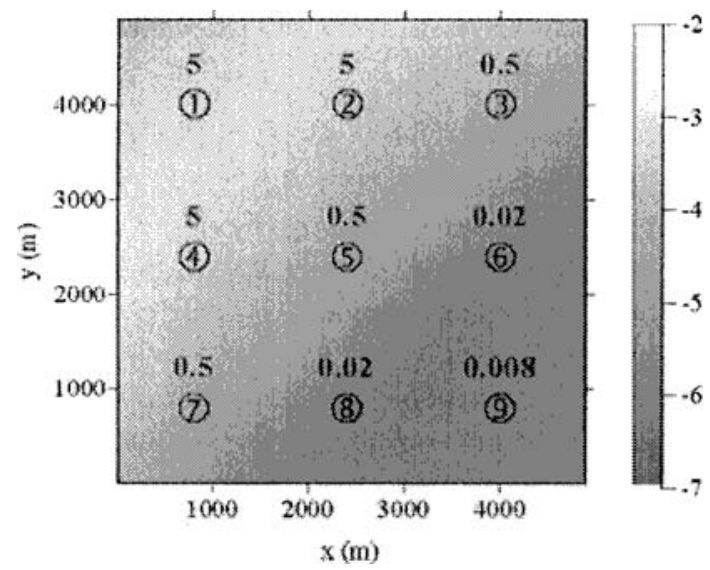

Figure 1. Reference $\log (T)$ field $\left(T\right.$ in $\left.\mathrm{m}^{2} / \mathrm{s}\right)$. Circled numbers show the positions of the abstraction wells; numbers above the labels show discharge rates in $\mathrm{L} / \mathrm{s}$.

well no. 9 negative transmissivities have been identified in a large region (the black area of the bottom plot of figure 2).
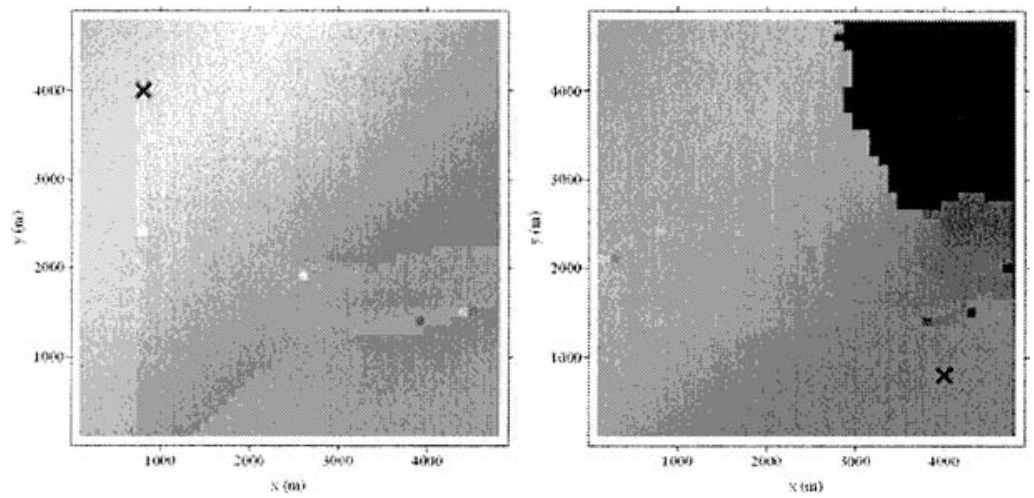

Figure 2. $\log (T)$ field identified with the standard DSM. The crosses denote the positions of the starting point. Gray scale is the same as for figure 1.

The results obtained when all the sets of data are used simultaneously, with the technique described in the previous section, are shown in figure 3 , again for the starting point at well no. 1 or 9 . These results show that this approach is very useful to reduce the dependence of the final solution on the starting point, which can be chosen almost everywhere without worsening the results of the DSM. 

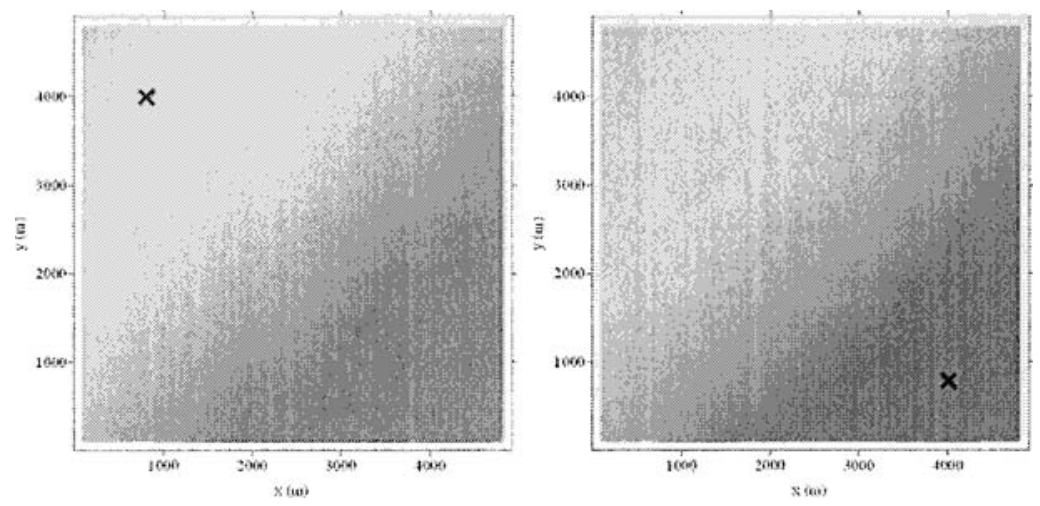

Figure 3. $\log (T)$ field identified with the modified DSM. The crosses denote the positions of the starting point. Gray scale is the same as for figure 1 .

\section{Conclusions and perspectives}

The new features of the DSM are very important, because they permit to limit the problems related to the choice of the starting point and to the availability of data sets which are independent throughout the whole domain.

Several perspectives are still open.

When $M$ sets of data are available, $M$ balance equations like (1) can be written, one for each data set, so that $A$ becomes a rectangular matrix with $M$ rows and two columns. In this case the vectors $\mathbf{a}$ and $\mathbf{b}$ can be computed with the least-squares technique. Numerical tests are going on to evaluate this alternative.

The numerical tests presented here show the importance of considering $\mu(A)$ for the computation of the vectors $\mathbf{a}$ and $\mathbf{b}$, which is the first stage of the DSM. Also the successive stage, the choice of the integration path, can be improved by considering different combinations of the three conditions introduced in this paper: the independence condition, the stability condition and the $\mu(A)$ condition.

Eventually, the method can be applied to transient data; in that case a large number of sets of data, corresponding to measurements at different times, could be available. The criteria for the estimation of the time derivative of the piezometric head and for the choice of the data sets to be used are of paramount importance.

\section{References}

[1] J. Carrera, S.P. Neuman. Estimation of aquifer parameters under transient and steady-state conditions: 1, maximum likelihood method incorporating prior information. Water Resour. 
Res. 22:199-210, 1986.

[2] G. Chavent. Analyse fonctionnelle et identification de coefficients répartis dans les équations aux dérivées partielles. Thése d'etat, Fac. des Science de Paris, 1971.

[3] Y. Emsellem, G. de Marsily. An automatic solution for the inverse problem. Water Resour. Res. 7:1264-1283, 1971.

[4] T.R. Ginn, J.H. Cushman, M.H. Houch. A continuous \{time inverse operator for groundwater and contaminant transport modeling: deterministic case. Water Resour. Res. 26:241$252,1990$.

[5] M. Giudici, G. Morossi, G. Parravicini, G. Ponzini. A new method for the identification of distributed transmissivities. Water Resour. Res. 31:1969-1988, 1995.

[6] M. Giudici, F. Delay, G. de Marsily, G. Parravicini, G. Ponzini, A. Rosazza. Discrete stability of the Differential System Method evaluated with geostatistical techniques. Stochastic Hydrol. and Hydraul. 12:191-204, 1998.

[7] S. Liu, T.-C. J. Yeh, R. Gardiner. Effectiveness of hydraulic tomography: Sandbox experiments Water Resour. Res. doi:10.1029/2001WR000338, 2002.

[8] R.W. Nelson. In place measurement of permeability in heterogeneous media, 1, Theory of a proposed method. J. Geophys. Res. 65:1753-1760, 1960.

[9] R.W. Nelson. In place measurement of permeability in heterogeneous media, 2, Experimental and computational considerations. J. Geophys. Res. 66:2469-2478, 1961.

[10] R.W. Nelson. Condition for determining areal permeability distribution by calculation. Soc. Pet. Eng. J. 2:223-224, 1962.

[11] G. Parravicini, M. Giudici, G. Morossi, G. Ponzini. Minimal a priori assignment in a direct method for determining phenomenological coefficients uniquely. Inverse Problems 11:611-629, 1995.

[12] G. Ponzini, A. Lozej. Identification of aquifer transmissivities: the comparison model method. Water Resour. Res. 18:597-622, 1982.

[13] G.R. Richter. An inverse problem for the steady state diffusion equation. SIAM J. Math. Anal. 41:210-221, 1981.

[14] B. Sagar, S. Yakowitz, L. Duckstein. A direct method for the identification of the parameters of dynamic non-homogeneous aquifers. Water Resour. Res. 11:563-570, 1975.

[15] S. Scarascia, G. Ponzini. An approximate solution for the inverse problem in hydraulics. L'Energia Elettrica 49:518-531, 1972.

[16] M.F. Snodgrass, P.K. Kitanidis. Transmissivity identification through multi-directional aquifer stimulation. Stochastic Hydrol. and Hydraul. 12:299-316, 1998.

[17] R. Vàzquez Gonzàlez, M. Giudici, G. Parravicini, G. Ponzini. The differential system method for the identification of transmissivity and storativity. Transport in Porous Media 26:339-371, 1997.

[18] W.-G. W. Yeh. Review of parameter identification procedures in groundwater hydrology: the inverse problem. Water Resour. Res. 22:95-108, 1986.

[19] T.-C. J. Yeh, S. Liu. Hydraulic tomography: Development of a new aquifer test method. Water Resour. Res. 36:2095-2105, 2000. 